\title{
The results of a third Gamma Knife procedure for recurrent trigeminal neuralgia
}

\author{
*Zachary J. Tempel, MD, ${ }^{1}$ Srinivas Chivukula, MD, ${ }^{2}$ Edward A. Monaco III, MD, PhD, ${ }^{1}$ \\ Greg Bowden, MD, ${ }^{1}$ Hideyuki Kano, MD, PhD, ${ }^{1}$ Ajay Niranjan, MD, MBA, ${ }^{1}$ Edward F. Chang, MD, ${ }^{3}$ \\ Penny K. Sneed, MD, ${ }^{4}$ Anthony M. Kaufmann, MD, BSc(Med), MSc, FRCSC, ${ }^{5}$ \\ Jason Sheehan, MD, PhD, ${ }^{6}$ David Mathieu, MD, FRCS(C), ${ }^{7}$ and L. Dade Lunsford, MD ${ }^{1}$
}

\begin{abstract}
1'Department of Neurological Surgery, University of Pittsburgh Medical Center; ${ }^{2}$ University of Pittsburgh School of Medicine, Pittsburgh, Pennsylvania; ${ }^{3}$ Departments of Neurological Surgery and ${ }^{4}$ Radiation Oncology, University of California, San Francisco, California; ${ }^{6}$ Department of Neurological Surgery, University of Virginia Health System, Charlottesville, Virginia; ${ }^{5}$ Department of Neurological Surgery, University of Manitoba Health Sciences Centre, Winnipeg, Manitoba; and 'Department of Neurological Surgery, Centre Hospitalier Universitaire de Sherbrooke, Quebec, Canada
\end{abstract}

\begin{abstract}
OBJECT Gamma Knife radiosurgery (GKRS) is the least invasive treatment option for medically refractory, intractable trigeminal neuralgia (TN) and is especially valuable for treating elderly, infirm patients or those on anticoagulation therapy. The authors reviewed pain outcomes and complications in TN patients who required 3 radiosurgical procedures for recurrent or persistent pain.
\end{abstract}

METHODS A retrospective review of all patients who underwent 3 GKRS procedures for TN at 4 participating centers of the North American Gamma Knife Consortium from 1995 to 2012 was performed. The Barrow Neurological Institute (BNI) pain score was used to evaluate pain outcomes.

RESULTS Seventeen patients were identified; 7 were male and 10 were female. The mean age at the time of last GKRS was 79.6 years (range 51.2-95.6 years). The TN was Type I in 16 patients and Type II in 1 patient. No patient suffered from multiple sclerosis. Eight patients (47.1\%) reported initial complete pain relief (BNI Score I) following their third GKRS and 8 others (47.1\%) experienced at least partial relief (BNI Scores II-IIIb). The average time to initial response was 2.9 months following the third GKRS. Although 3 patients (17.6\%) developed new facial sensory dysfunction following primary GKRS and 2 patients (11.8\%) experienced new or worsening sensory disturbance following the second GKRS, no patient sustained additional sensory disturbances after the third procedure. At a mean follow-up of 22.9 months following the third GKRS, 6 patients (35.3\%) reported continued Score I complete pain relief, while 7 others (41.2\%) reported pain improvement (BNI Scores II-IIIb). Four patients (23.5\%) suffered recurrent TN following the third procedure at a mean interval of 19.1 months.

CONCLUSIONS A third GKRS resulted in pain reduction with a low risk of additional complications in most patients with medically refractory and recurrent, intractable TN. In patients unsuitable for other microsurgical or percutaneous strategies, especially those receiving long-term oral anticoagulation or antiplatelet agents, GKRS repeated for a third time was a satisfactory, low risk option.

http://thejns.org/doi/abs/10.3171/2014.9.JNS132779

KEY WORDS Gamma Knife; microvascular decompression; trigeminal neuralgia; refractory; stereotactic radiosurgery; functional neurosurgery

$\mathrm{T}$ RIGEMINAL neuralgia (TN) typically manifests as intense paroxysmal, brief, electric, or stabbing unilateral facial pain along the dermatomal distribution of one or more branches of the trigeminal nerve. It is often triggered by cutaneous light touch stimulation. ${ }^{10,11}$ Several variants of TN are appreciated, and management options vary. Surgical options for medically refractory TN include microvascular decompression (MVD) as well

ABBREVIATIONS BNI = Barrow Neurological Institute; GKRS = Gamma Knife radiosurgery; MVD = microvascular decompression; NAGKC = North American Gamma Knife Consortium; PRGR = percutaneous retrogasserian glycerol rhizotomy; TN = trigeminal neuralgia.

SUBMITTED December 15, 2013. ACCEPTED September 23, 2014.

INCLUDE WHEN CITING Published online October 31, 2014; DOI: 10.3171/2014.9.JNS132779.

DISCLOSURE Dr. Lunsford is a consultant for and a stockholder with AB Elekta. The remaining authors report no conflicts of interest concerning the materials or methods used in this study or the findings specified in this paper.

* Drs. Tempel and Chivukula contributed equally to this work. 
as percutaneous ablative procedures such as radiofrequency rhizotomy, glycerol rhizotomy, balloon compression, and alcohol blocks. ${ }^{49,50}$ Because of its less invasive nature, Gamma Knife radiosurgery (GKRS) has become a well-established alternative surgical option with a low risk of complications.

Initial pain relief after GKRS has been related in part to the radiation dose delivered to the postganglionic intracranial nerve. ${ }^{6,50}$ As the total maximal dose increases, a higher number of patients note postprocedural trigeminal nerve dysfunction ranging from mild facial numbness or paresthesias to annoying deafferentation sequelae such as dysesthesias. ${ }^{6,22,47}$ Regardless of the dose delivered, the benefit of radiosurgery may decline over time and may lead to symptom recurrence. Among management options for recurrent medically refractory trigeminal neuralgia (TN), GKRS is often appealing in patients with advanced age or medical comorbidities, especially for those patients on a long-term regimen of anticoagulation or antiplatelet therapy.

The rate of initial pain relief among patients undergoing primary GKRS for idiopathic TN ranges from $78 \%$ to $94 \%$, with between $32 \%$ and $81 \%$ of patients experiencing complete pain relief. . $8,30,42$ At a median of approximately 2 years, between $25 \%$ and $52 \%$ of these patients can develop recurrent TN..$^{15,27,46}$ Such patients have been found to benefit from repeat GKRS, with success rates of between $62 \%$ and $95 \%$ at a median 32-month follow-up. $1,9,13,16,18,19,24,34,36,45$ In this report, we describe our experience in a small cohort of patients who ultimately underwent 3 GKRS procedures because of the recurrence of medically intractable pain. We analyze the outcomes in such patients, including the extent and duration of pain relief.

\section{Methods \\ Patient Selection}

The 19 participating centers in the North American Gamma Knife Consortium (NAGKC) were polled and were queried whether they had experience in the use of the Leksell Gamma Knife for recurrent TN. Four academic medical centers reported that they had patients who underwent GKRS at least 3 times for refractory pain: the University of Pittsburgh Medical Center (9 patients), the University of California at San Francisco (3 patients), the University of Virginia (3 patients), the University of Manitoba (1 patient), and L'Université de Sherbrooke (1 patient). Each institution received institutional review board approval for retrospective clinical outcomes studies, following which the records of all patients who underwent 3 radiosurgical procedures for TN were assessed, stripped of patient identifying information, and submitted to the NAGKC clinical coordinating center. This process is similar to that used in other studies previously conducted by the NAGKC. ${ }^{21}$ The 17 patients included in this study underwent GKRS at their respective institutions between 1995 and 2012. Pre- and post-GKRS clinical and radiographic data were assessed at a median follow-up period of 22.9 months (range 3.0-60.0 months) via outpatient visits or phone interviews conducted by clinical staff at each institution.

All patients included in the study underwent GKRS 3 times for $\mathrm{TN}$ that was recurrent despite ongoing medical management and prior surgical procedures. Comprehensive but individualized medical therapies included agents such as carbamazepine, oxcarbazepine, phenytoin, clonazepam, lorazepam, gabapentin, baclofen, and also serotonin reuptake inhibitors in selected patients. Although patients in this study were drawn from 4 different centers across North America, the data collection process is consistent and therefore conducive to research analyses. Each center individually performs its radiosurgical operations on patients deemed fit, in this case for a third GKRS treatment for intractable, recurrent TN. Patients are selfreferred or referred by their primary health care providers, other providers, or other centers. All centers routinely catalog the patients that go through their practice, such that their data can be easily recalled upon request, coordinated by the NAGKC clinical coordinating center, headquartered at the University of Pittsburgh Medical Center. The data are prospectively collected but retrospectively analyzed in response to a query for data corresponding to patients who met the inclusion and exclusion criteria of the study.

The demographics of our series are outlined in Table 1. There were 7 male and 10 female patients with a mean age of 79.6 years (range 51.2-95.6 years) at the time of the third GKRS procedure. Nine patients underwent a total of 18 surgical procedures prior to their first GKRS. These included 11 percutaneous retrogasserian glycerol rhizotomies (PRGRs) in 6 patients, microsvascular decompression in 3 patients, and 4 percutaneous radiofrequency thermal rhizotomies in 1 patient. One patient underwent 2 PRGRs and 1 MVD. Five of the 9 patients underwent more than one surgical procedure prior to the first GKRS. The pain distribution at the time of first GKRS was found most commonly to be along the V2 and V3 distributions (7 patients, $41.2 \%$ ) and right-sided (12 patients, 70.6\%). Four patients $(23.5 \%)$ had facial sensory dysfunction prior to the first GKRS, manifested as hypesthesia, hypalgesia, or deafferentiation pain such as burning pain. Finally, 16 of 17 patients included in this study had Type I pain by the classification of Burchiel. ${ }^{4}$ These patients described their pain as sharp, lancinating, and shock like with painfree intervals. ${ }^{34}$ One patient had Type II pain, described as mostly constant, aching or stabbing pain. ${ }^{43}$ Three patients had Type I pain suspected to be related to vascular compression as seen on brain imaging.

\section{Radiosurgical Procedures}

Various models of the Gamma Knife (models U, B, $\mathrm{C}, 4 \mathrm{C}$, and Perfexion, Elekta $\mathrm{AB}$ ) were used during this study. The radiosurgical procedure has been previously described. ${ }^{21,34}$ After application of a Leksell stereotactic frame (Elekta Instruments) under local anesthesia and intravenous conscious sedation, all patients underwent stereotactic MRI to identify the trigeminal nerve. MRI was performed by the use of contrast-enhanced, short repetition time sequences, and axial volume acquisitions divided into 1-mm slices. Additional axial long relaxation time MRI sequences were obtained in cases in which the trigeminal nerve was difficult to identify, usually due to prior surgery. A team consisting of a neurosurgeon, ra- 
TABLE 1. Patient demographics

\begin{tabular}{|c|c|}
\hline Characteristic & Value* $^{*}$ \\
\hline Total no. of patients & 17 \\
\hline Male & $7(41.2)$ \\
\hline Female & $10(58.8)$ \\
\hline \multicolumn{2}{|l|}{ Age at last GKRS (yrs) } \\
\hline Mean & 79.6 \\
\hline Range & $51.2-95.6$ \\
\hline \multicolumn{2}{|l|}{ Pain distribution } \\
\hline $\mathrm{V} 1$ & $1(5.9)$ \\
\hline V2 & $4(23.5)$ \\
\hline V3 & $3(17.6)$ \\
\hline V1 \& V2 & 0 \\
\hline V1 \& V3 & 0 \\
\hline V2 \& V3 & $7(41.2)$ \\
\hline $\mathrm{V} 1, \mathrm{~V} 2, \& \mathrm{~V} 3$ & $2(11.8)$ \\
\hline Right & $12(70.6)$ \\
\hline Left & $5(29.4)$ \\
\hline \multicolumn{2}{|l|}{ Preexisting facial sensory dysfunction } \\
\hline Hypesthesia & $2(11.8)$ \\
\hline Hypalgesia & $1(5.9)$ \\
\hline Burning pain & $1(5.9)$ \\
\hline \multicolumn{2}{|l|}{ Prior operation } \\
\hline Total no.† & 18 \\
\hline PRGR & $11(61.1)$ \\
\hline MVD & $3(16.7)$ \\
\hline RFL & $4(22.2)$ \\
\hline \multicolumn{2}{|l|}{ Trigeminal neuralgia } \\
\hline Type I & 16 \\
\hline Type II & 1 \\
\hline Symptomatic trigeminal neuralgia & 0 \\
\hline Vascular compression associated & 3 \\
\hline Atypical facial pain & 0 \\
\hline
\end{tabular}

$\mathrm{RFL}=$ radiofrequency lesioning.

* Values are number (\%) unless indicated otherwise.

$\dagger$ Percentages are based on the number of prior operations.

diation oncologist, and medical physicist performed dose selection and treatment planning.

A single 4-mm isocenter was used in all patients at the first radiosurgical procedure for targeting the trigeminal nerve. The trigeminal target was selected 3-4 $\mathrm{mm}$ anterior to its junction with the lateral pons. At the second radiosurgical procedure, the target was usually placed anterior to the first target for an overlap of the 2 radiosurgical volumes by approximately $50 \%$. The target for the third radiosurgical procedure was typically distal to the first and second targets, but their overlap varied and depended on the total pons-to-gasserian ganglion distance in each patient. This process ensured less radiation to the brainstem at each subsequent radiosurgical procedure, while providing additional radiation along the trigeminal nerve more proximal to the gasserian ganglion. The median maximum target dose was 80 Gy (range 70-85 Gy) for the first radiosurgical procedure, 70 Gy (range 40-80
Gy) for the second, and 70 Gy (range 40-80 Gy) for the third procedure. The median cumulative target dose was 210 Gy (range 150-240 Gy).

\section{Follow-Up Evaluations}

Follow-up information was obtained from the patients, their families, or their referring physicians. Data pertaining to the degree of pain relief, latency interval to pain relief, the need for further surgical procedures or medication, and new symptoms such as facial sensory dysfunction were obtained. The Barrow Neurological Institute (BNI) pain intensity criteria were used to grade the pain outcome for all patients in this study. Specifically, Score I (no pain, no medication); Score II (occasional pain, not requiring medication); Score IIIa (no pain, but continued medication); Score IIIb (pain controlled with medication); Score IV (pain improved, but not adequately controlled with medication); and Score V (persistent inadequately controlled). ${ }^{43}$ It is to be noted that although the original score developed by Rogers et al. ${ }^{43}$ only contained a score of III, we utilized a modification of this score to include 2 subcategories, IIIa and IIIb, defined as presented herein. For the purposes of this report, we defined BNI Score I as complete relief, BNI Scores II-IIIb as incomplete relief, and BNI Scores IV and V as no significant relief. BNI Scores I-IIIb were considered as adequate pain relief.

\section{Data and Analyses}

Patient demographics and outcomes were analyzed using descriptive statistics. The rates of initial pain improvement and the maintenance of pain relief among those showing initial response were analyzed by the productlimit method of Kaplan and Meier. A p value $<0.05$ was considered statistically significant. Data were collected using Excel (Microsoft Corp.) and were analyzed using SPSS (version 20.0, IBM).

\section{Results \\ Pain Response}

Table 2 details the treatment data for all 17 patients who underwent 3 GKRS procedures for their TN. Before their first procedure 12 patients had BNI Score V pain and 5 had BNI Score IV pain. The initial response was complete relief in 6 patients (35.3\%) following the first GKRS, in 8 patients (47.1\%) following the second GKRS, and in 8 patients $(47.1 \%)$ following the last GKRS. Only 1 patient did not obtain any period of pain relief following the third GKRS. Neither the rates of complete relief following GKRS nor the time to pain recurrence was found to be statistically different for subsequent GKRS procedures compared with the first GKRS. The mean time to recurrence after each radiosurgical procedure ranged from 19.1 to 34.8 months. The average interval between the first and the second radiosurgical procedures (34.3 months) was shorter than between the second and the third procedures (47.1 months).

Between the first and the second GKRS, 3 patients underwent 3 alternate surgical procedures that included 1 PRGR in each of 2 patients and a single MVD in the third patient. Three different patients underwent other surgical 
TABLE 2. Treatment data for patients who underwent triple GKSR for trigeminal neuralgia

\begin{tabular}{|c|c|c|c|c|}
\hline \multirow[b]{2}{*}{ Parameter } & \multicolumn{4}{|c|}{ No. of Patients (\%) } \\
\hline & Initial Presentation & GKSR 1 & GKSR 2 & GKSR 3 \\
\hline Total no. of patients & 17 & 17 & 17 & 17 \\
\hline \multicolumn{5}{|l|}{ BNI pain score } \\
\hline I & 0 & $6(35.3)$ & $8(47.1)$ & $8(47.1)$ \\
\hline II & 0 & $3(17.6)$ & $3(17.6)$ & $3(17.6)$ \\
\hline Illa & 0 & $4(23.5)$ & $4(23.5)$ & $5(29.4)$ \\
\hline IIlb & 0 & $4(23.5)$ & $2(11.8)$ & 0 \\
\hline IV & $5(29.4)$ & 0 & 0 & $1(5.9)$ \\
\hline V & $12(70.6)$ & 0 & 0 & 0 \\
\hline \multicolumn{5}{|l|}{ Maximum pain relief } \\
\hline Complete (BNI pain score I) & NA & $6(35.3)$ & $8(47.1)$ & $8(47.1)$ \\
\hline Incomplete (BNI pain score II-IIIb) & NA & $11(64.7)$ & $9(52.9)$ & $8(47.1)$ \\
\hline \multicolumn{5}{|l|}{ Time to pain recurrence (mos) } \\
\hline Mean & & 22.1 & 34.8 & 19.1 \\
\hline Range & & $1.0-84.8$ & $3.0-72.0$ & $2.1-60.0$ \\
\hline \multicolumn{5}{|l|}{ Time to subsequent GKRS (mos) } \\
\hline Mean & & & & NA \\
\hline Range & & 34.3 & 47.1 & NA \\
\hline Maximum treatment dose (Gy) & & $3.5-91.9$ & $16.8-104.2$ & \\
\hline Mean & NA & 79.7 & 65.9 & 62.9 \\
\hline Range & NA & $70-85$ & $40-80$ & $40-80$ \\
\hline \multicolumn{5}{|l|}{ Cumulative dose } \\
\hline Mean & NA & 79.7 & 145.6 & 208.5 \\
\hline Range & NA & $70-85$ & $110-160$ & $150-240$ \\
\hline \multicolumn{5}{|l|}{ Prior or interval surgery } \\
\hline PRGR & 11 & 2 & $4^{*}$ & 1 \\
\hline MVD & 3 & 1 & 0 & 0 \\
\hline Other & 4 & 0 & 0 & 2 \\
\hline \multicolumn{5}{|l|}{ Time to/from GKRS (mos) } \\
\hline Mean & 20.1 & 17.3 & 40.6 & 10.0 \\
\hline Range & $1.0-68.6$ & $11.2-25.0$ & $30.0-56.1$ & $3.0-17.0$ \\
\hline
\end{tabular}

procedures (all PRGR) between the second and the third GKRS; 2 patients underwent 1 PRGR each, while PRGR was performed twice in the third patient prior to the third GKRS. Two patients underwent additional surgical procedures after the last GKRS -2 infraorbital nerve sectioning procedures in a single patient and a single PRGR in the other.

As shown in Table 2, in the initial period following the first GKRS, 6 patients (35.3\%) experienced complete pain relief. Three others (17.6\%) reported a BNI Score II response, and 4 patients $(23.5 \%)$ reported each of BNI Score IIIa and IIIb responses. All patients responded to treatment. Following the second radiosurgical procedure, complete relief was reported by 8 patients $(47.1 \%)$. Three patients $(17.6 \%)$ reported a BNI Score II response, while 6 others experienced at least adequate relief, with a BNI Score IIIa response in 4 patients $(23.5 \%)$ and Score IIIb in 2 patients (11.8\%). Following the third GKRS and at a mean latency interval of 2.9 weeks, 8 patients (47.1\%) reported an initial response of complete relief, and an additional 8 patients experienced adequate relief ( 3 patients to a BNI score of II and 5 to a BNI score of IIIa). A single patient did not respond (BNI Score IV) to treatment. Finally, at the last evaluation (mean follow-up, 22.9 months; range 3.0-60.0 months) after the third procedure, $6 \mathrm{pa}-$ tients $(35.3 \%)$ reported BNI Score I pain relief, while 7 others (47.1\%) reported BNI scores of IIIb or better. Four patients $(23.5 \%)$ had recurrent pain with a BNI pain score of IV. Treatment outcomes are shown in Table 3. The mean time to initial response, or latency interval, did not significantly differ among the 3 radiosurgical procedures, averaging 4.1 months after the first GKRS and a consistent 2.9 months after the second and third radiosurgical procedures. The statistical similarity among the 3 GKRS procedures is shown in Fig. 1, which illustrates that the probability of experiencing initial pain relief as a function 
TABLE 3. Outcomes in patients who underwent triple GKRS for trigeminal neuralgia

\begin{tabular}{lccc}
\hline \multirow{2}{*}{ Parameter } & \multicolumn{3}{c}{ No. of Patients (\%) } \\
\cline { 2 - 4 } & GKSR 1 & GKSR 2 & GKSR 3 \\
\hline Time to initial response (wks) & & & \\
\hline Mean & 4.1 & 2.9 & 2.9 \\
\hline Range & $0.5-20.9$ & $0.5-8.0$ & $0.5-6.7$ \\
\hline New facial sensory dysfunction & $3(17.6)$ & $2(11.8)$ & 0 \\
\hline Temporary & 0 & $1(5.9)$ & 0 \\
\hline Permanent & $3(17.6)$ & $1(5.9)$ & 0 \\
\hline BNI pain score at last evaluation & & & \\
\hline I & NA & NA & $6(35.3)$ \\
\hline II & NA & NA & 0 \\
\hline IIla & NA & NA & $4(23.5)$ \\
\hline IIlb & NA & NA & $3(17.6)$ \\
\hline IV & NA & NA & $4(23.5)$ \\
\hline V & NA & NA & 0 \\
\hline Follow-up (mos) & \multicolumn{2}{c}{} \\
\hline Mean & NA & NA & 22.9 \\
\hline Range & NA & NA & $3.0-60.0$ \\
\hline
\end{tabular}

of time from radiosurgery did not differ among the three procedures. Figure 2 depicts the probability of maintenance of pain relief after GKRS.

\section{Complications}

As shown in Table 3, 3 patients reported the development of new trigeminal sensory dysfunction, or worsening of preexisting dysfunction, after the first GKRS procedure, and 2 patients after the second GKRS; 4 of the 5 patients described persistent sensory loss. Among the

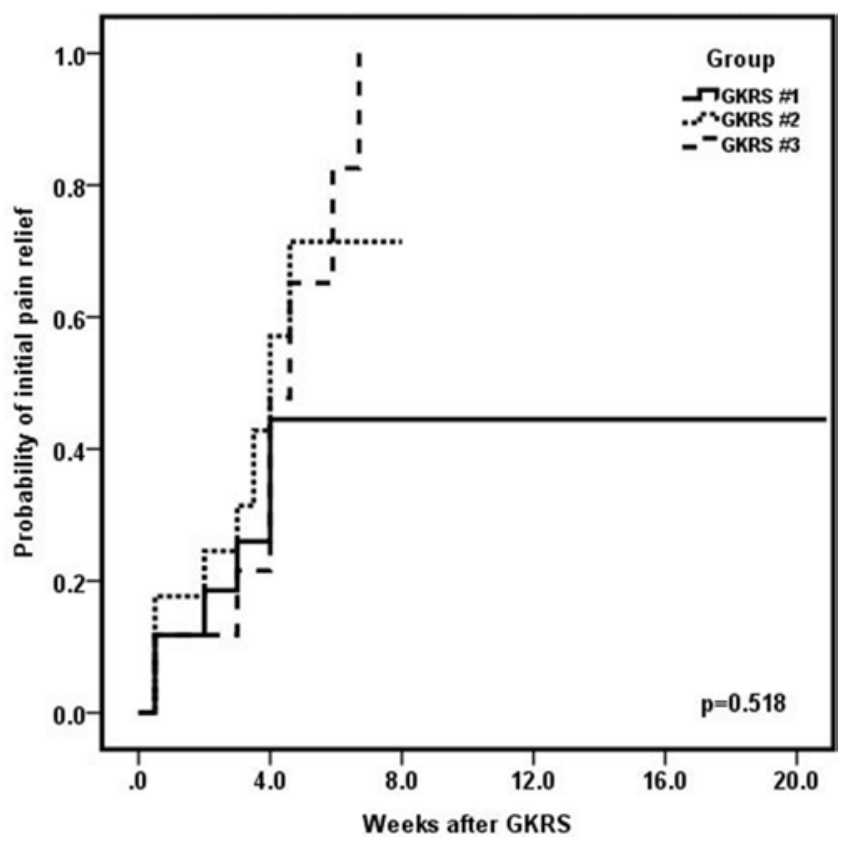

FIG. 1. Kaplan-Meier estimates of the probability of initial pain relief following triple GKRS for TN (BNI Scores I-IIlb).

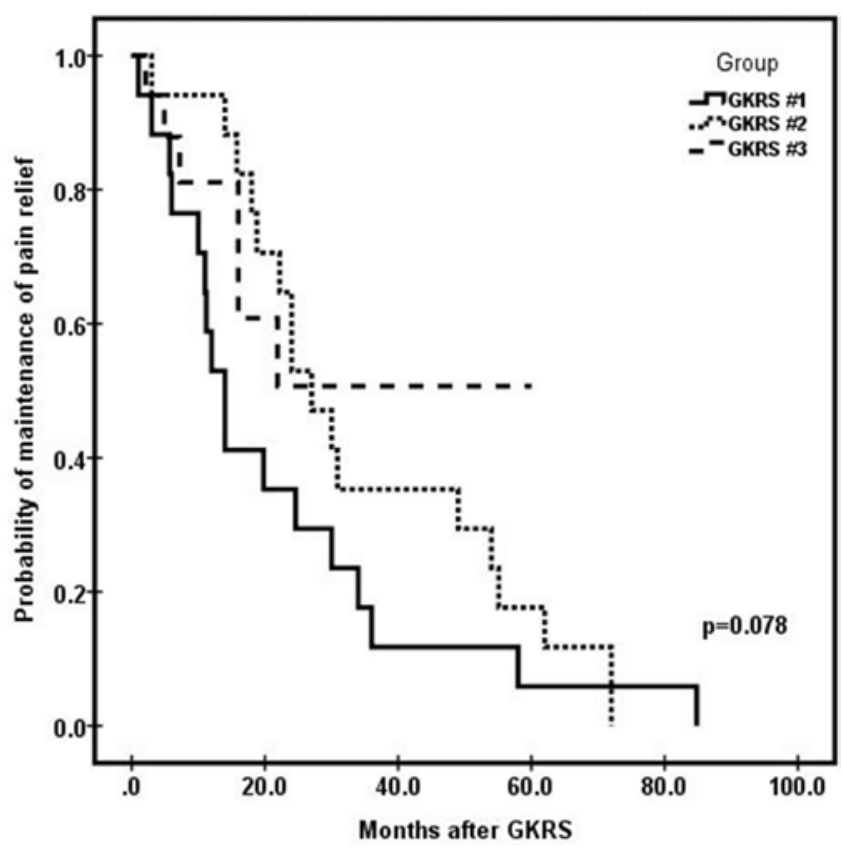

FIG. 2. Kaplan-Meier estimates of maintenance of pain relief following triple GKRS for TN.

3 patients in the former group, 1 patient presented with TN along the right-sided V3 distribution associated with hypesthesia and hypalgesia along the same dermatome; within 3 months after the last procedure the patient developed additional hypesthesia and hypalgesia along the right V2 dermatome. While his TN pain along the V3 distribution improved, the sensory dysfunction along this dermatome did not change. A second patient developed hypesthesia along the right-sided V1 and V2 distributions after undergoing GKRS for right-sided V3 pain; this patient had presented initially with numbness along the V3 dermatome (as much as 50\%), which did not change postprocedurally. Finally, a third patient experienced worsening of preexisting hypesthesia after undergoing GKRS for right-sided $\mathrm{TN}$ primarily along the V2 dermatome. In all 3 of these patients, the trigeminal sensory dysfunction was persistent.

Following the second radiosurgical procedure, 2 additional patients reported sensory complications. Both of these patients underwent GKRS for TN pain along the right-sided V2 and V3 dermatomes. Following the second GKRS, they were noted to be $10 \%$ hypesthetic along all dermatomes of their right-sided trigeminal nerve by the 1st month of follow-up. In one of these 2 patients the sensory dysfunction resolved spontaneously by the 2nd month of follow-up, while it persisted in the other. No sensory complications were noted following the third radiosurgical procedure. It is interesting to note that despite the development of trigeminal sensory dysfunction including the V1 dermatome in some patients, no patient in our series developed corneal complications such as abrasions, ulcerations, or other keratopathy. A single patient was noted after the third GKRS to have developed scar tissue at the trigeminal root entry zone that was found on follow-up MRI, but this was not associated with evident symptomatology. This 
patient had, however, undergone 2 PRGR procedures prior to the third GKRS; it is likely that the scar tissue formation reflected fibrosis in response to the repeated injection and is not a response to the GKRS itself.

\section{Discussion}

The efficacy of an initial GKRS in achieving pain relief from TN has been well described. . $^{2,5,6,8,12,20,30,39,41,42} \mathrm{Un}$ fortunately, all current surgical procedures are associated with varying risks of pain relapse. Han et al. noted that despite an initial rate of pain relief of more than $90 \%$ after GKRS for idiopathic TN, 52\% of patients experienced a recurrence between 2 and 56 months post-GKRS. $.^{15} \mathrm{Nu}-$ merous reports have detailed success with repeat GKRS for recurrent $\mathrm{TN}$, especially in patients who demonstrate a good response after initial GKRS. More than $60 \%$ of patients undergoing repeat GKRS experience at least 50\% improvement in pain. ${ }^{1,9,13,16,18,19,24,34,36,45}$ Yet $25 \%$ of patients experienced recurrent pain (BNI Score IV or V) at a median of 17 months following a repeat GKRS. ${ }^{26,34}$ We hypothesized that there exists a subset of patients who may benefit from a third radiosurgical procedure, predicted by their prior favorable response to GKRS. TN is frequent in the elderly population; many of these patients are on a regimen of anticoagulation and/or antiplatelet therapy that poses bleeding risks to invasive microsurgical or percutaneous procedures. Additionally, many such patients have coexisting morbidity from associated medical conditions. In such a population minimally invasive procedures are advantageous for $\mathrm{TN}$ patients intolerant to, or whose pain is ineffectively controlled by, medication.

\section{Repeat GKRS for TN}

Data on multiply repeated GKRS for TN in the literature to date are scant. Analyses of the safety and efficacy of more than 1 radiosurgical procedure for $\mathrm{TN}$ have thus far been limited to reports of a single repeated GKRS procedure. Therefore, the efficacy and safety of a third GKRS procedure can only be compared with those data for single, and single repeat, procedures. In a recent analysis of 503 patients with idiopathic, medically refractory TN, Kondziolka et al. reported that $80 \%$ of patients experienced satisfactory pain control (BNI Scores I-IIIb) at 1 year following primary GKRS and that $46 \%$ maintained this relief at 5 years after GKRS. ${ }^{27}$ Villavicencio and colleagues reported an initial pain relief not requiring medication (BNI Scores I and II) in 67\% of 95 patients who underwent first-time CyberKnife radiosurgery for medically refractory, idiopathic TN, $50 \%$ of whom maintained complete relief ( $>90 \%$ pain relief) at 2 years after radiosurgery. ${ }^{29,48}$

Rates of initial pain relief reported in the literature for single repeat GKRS in TN patients are similar. ${ }^{1,9,13,16,18,19,24,34,36,45}$ In a large recent series, Park et al. described their experience with repeat GKRS for recurrent TN in 119 patients at a median of 26 months after primary GKRS and noted that $87 \%$ of patients achieved initial pain relief (BNI Scores I-IIIb), $88 \%$ of whom maintained relief at 1 year, and $70 \%$ of whom maintained relief at 3 years after repeat GKRS. ${ }^{34}$ Aubuchon et al. and
Gellner et al., in separate analyses, also reported similar response rates for repeat GKRS for recurrent TN; $81 \%$ of 37 patients who underwent repeat GKRS after a median interval of 15 months from primary GKRS experienced > $50 \%$ pain relief in the former study, and 16 of 22 patients in the latter study experienced initial reduction in their TN pain to BNI Scores I-IIIb (from BNI Score IV or V) following repeat GKRS at a median of 19 months following primary GKRS. ${ }^{1,13}$ In our own series, all patients experienced significant pain relief (BNI Scores I-IIIb) after the first and second GKRS procedures and underwent a third GKRS because of recurrent pain in patients and because they were considered high risk for more invasive $\mathrm{TN}$ surgical procedures.

The initial repeat GKRS for recurrent TN in our $17 \mathrm{pa}-$ tients was performed at a median of 35.2 months following primary GKRS, and the third GKRS at a median of 38.1 months after initial repeat GKRS. While these intervals are significantly longer than those reported for single repeat GKRS, it must be remembered that all patients in our patient sample experienced at least adequate pain relief after both the initial and the second radiosurgical procedures and therefore are not representative of the general population. Sixteen of 17 of patients in our series, or 94.1\%, experienced initial pain relief (BNI Scores I-IIIb) following the third GKRS; 8 (47.1\%) were completely relieved of their pain. Although not statistically different from the rates of initial pain relief reported with primary and initial repeat GKRS for TN, this high initial success rate likely reflects a patient sample biased toward positive outcomes, supporting our working hypothesis that those patients with a prior favorable response to GKRS might benefit from a third GKRS for their recurrent, medically refractory TN. ${ }^{8,16,18,30,34,36,42,45}$

The latency to pain relief among patients undergoing initial GKRS for TN has been variously noted to range from 1 to 2 months. ${ }^{8,16,18,27}$ Kondziolka et al. reported that $89 \%$ of their patients responded to treatment at a median of 1 month after initial GKRS; Villavicencio and colleagues observed a median time to pain relief of 2 weeks following initial CyberKnife radiosurgery. ${ }^{27,29,48}$ There is consensus that those patients with the fewest alternate surgical treatments prior to initial radiosurgery tend to experience the shortest latency intervals to pain relief following radiosurgery. ${ }^{27,29,34}$ Among patients undergoing single repeat GKRS, Park et al. noted a median latency interval of 6 weeks, additionally hypothesizing that patients who experienced favorable responses to primary GKRS may experience shorted latency intervals after repeat GKRS. ${ }^{34}$ It appears that our series supports this latter proposition; the median latency interval to pain relief was 4.1 weeks for the first radiosurgical procedure but 2.9 weeks for each of the repeat radiosurgical procedures. Furthermore, it appears that those patients with the greatest initial pain relief (lower BNI scores) after initial GKRS may tend to experience shorter subsequent latency intervals, although our sample is not statistically powered to validate this trend.

\section{The Effect of Other Surgical Procedures on Outcomes}

The impact of interval alternate surgical procedures 
between GKRS procedures for TN has not been well established. ${ }^{3,18,40,46}$ Among patients undergoing primary PRGR for classic TN, rates of initial pain relief of between $79 \%$ and $92 \%$ have been reported, with $50 \%$ of patients pain free after 4 years. ${ }^{7,17,32,35}$ Among patients with recurrent TN after PRGR who undergo GKRS, repeat PRGR has been reported to be associated with faster pain relief than repeat GKRS (median time to pain relief $<24$ hours), but shorter duration of pain relief, greater perioperative morbidity, and a higher failure rate than GKRS. ${ }^{31,32}$ One patient in our series underwent PRGR 17 months following the third GKRS. Initial pain relief after radiofrequency rhizotomy for TN has also been reported to occur in a high percentage of patients, between $97 \%$ and $100 \%$, with $50 \%$ maintaining relief at long-term follow-up. ${ }^{23,28,32,33}$ As with repeat PRGR, however, the efficacy of repeat radiofrequency rhizotomy is dramatically lower than that with repeat GKRS. Recurrence rates of even 100\% have been reported in less than 2 years following repeat PRGR. ${ }^{23,28,33}$ It appears in our series that outcomes following the third GKRS are influenced more by prior response to GKRS rather than by a history of additional surgical procedures performed in this patient cohort.

\section{Trigeminal Sensory Dysfunction After Triple GKRS}

The greatest potential risk of repeat radiosurgery procedures is cumulative trigeminal sensory dysfunction. After primary GKRS for TN, some degree of trigeminal dysfunction has been reported to occur in between $6 \%$ and $54 \%$ of patients. ${ }^{6,15,27,34,38,39,41,46}$ In $11 \%-74 \%$ of these patients there is worsening of preexisting trigeminal disturbance or new onset of dysfunction following repeat radiosurgery for TN. $1,9,13,16,18,21,24,27,34,43,45$ Most patients with TN typically report satisfactory pain relief without bothersome sensory dysfunction following the radiosurgical procedure. Yet several studies have correlated trigeminal sensory loss following GKRS with improved pain control. ${ }^{21,24,27,34}$

In our series, 3 patients (17.6\%) developed trigeminal sensory dysfunction following the primary GKRS. These included hypesthesia in 3 patients and hypalgesia in 1 patient. One of the 2 patients with new hypesthesia experienced permanent worsening of the dysfunction after the second GKRS. One additional patient reported temporary hypesthesia after the second GKRS that resolved within 2 months of the procedure, and a second patient developed persistent hypesthesia. It is interesting to note that no patient in our series experienced new or worsening of a trigeminal sensory disturbance following the third GKRS. As has been mentioned, higher doses at individual treatments can yield better pain relief but with more sensory disturbance. ${ }^{6,22,27,29,34,47,48}$ However, it may be that cumulative doses need to be much higher than reported here to produce dramatically higher rates of sensory disturbance.

Our record of trigeminal sensory disturbances as complications of radiosurgery merits further explanation regarding the method of their follow-up. Side effects postradiosurgery are primarily sensory in nature; in addition, sensory side effects are often not patient reported but come to notice only upon careful examination by a skilled health care practitioner. Proper follow-up is therefore cru- cial to an accurate record of outcomes, especially those pertaining to sensory function and following radiosurgery. All patients in our series routinely undergo followup in a neurosurgical clinic with providers who perform the neurological examination in a consistent manner and religiously document their findings. No patient in our series was lost to follow-up; therefore, all data pertaining to sensory function were obtained from in clinic visits. If patients were found to have sensory complications, their severity was monitored and follow-up information was obtained via phone calls to patients or reports from their primary care providers.

Park et al. recently reported that the maximum cumulative lateral pontine edge dose predicted the development of facial sensory dysfunction, noting $44 \mathrm{~Gy}$ of cumulative brainstem dose as the cutoff. ${ }^{34}$ In another study, Huang et al. observed that 115 Gy of cumulative maximum target dose increased the chance that patients developed new sensory symptoms. ${ }^{19,20}$ In yet another study, Aubuchon et al. reported $108.5 \mathrm{~Gy}$ as the threshold beyond which sensory disturbances from GKRS may be realistically expected. ${ }^{1}$ In this context, the lack of sensory dysfunction among our patients may relate to target selection at each GKRS; moving the target anteriorly (closer to the gasserian ganglion) with each subsequent radiosurgical procedure limits the dose that is received by the brainstem. In theory it might, however, increase sensory loss by targeting the cell bodies located in the gasserian ganglion. It is likely that target location, dose, and the time since prior radiosurgical procedure are the key variables to consider in optimizing GKRS for TN.

\section{Maintenance}

Dose-selection and target positioning for primary GKRS for TN have become increasingly uniform since the first multiinstitutional study by Kondziolka et al., yet controversy surrounds that for repeat GKRS. ${ }^{26}$ Between 70 and 90 Gy is generally accepted as optimal for pain control at the first radiosurgical procedure; doses of 90 Gy are associated with faster onset of pain relief and perhaps longer duration of pain relief. ${ }^{14,25}$ Historically, most authors have recommended a reduced dose at the second radiosurgical procedure, for a cumulative dose of 115-170 Gy. $3,16,18,19,36,37,44-46$ In a recent series of 119 patients undergoing repeat GKRS for recurrent TN, Park et al. reported using a median cumulative dose of 145 Gy (range 120 170 Gy) and obtaining an $87 \%$ initial pain relief $(>50 \%$ pain relief or BNI Scores I-IIIb). ${ }^{34}$ The pain relief was maintained in $69.8 \%$ of these patients at 3 years following GKRS and in $44.2 \%$ at 5 years. ${ }^{14}$

As stated earlier, in our series, a single 4-mm isocenter was used in all patients at the first radiosurgical procedure for targeting the trigeminal nerve. The trigeminal target was selected 3-4 $\mathrm{mm}$ anterior to its junction with the lateral pons. To avoid excessive radiation to the brainstem and to minimize adverse effects, the target at the second radiosurgical procedure was usually placed anterior to the first target for an overlap of the 2 radiosurgical volumes by approximately $50 \%$. The target for the third radiosurgical procedure was typically distal to the first and second targets, but their overlap varied and depended 
on the total pons-to-gasserian ganglion distance in each patient. This process ensured less radiation to the brainstem at each subsequent radiosurgical procedure, while providing additional radiation along the trigeminal nerve more proximal to the gasserian ganglion. The median maximum target dose was 80 Gy (range $70-85$ Gy) for the first radiosurgical procedure, 70 Gy (range 40-80 Gy) for the second, and 70 Gy (range 40-80 Gy) for the third procedure. The median cumulative target dose was 210 Gy (range 150-240 Gy).

Overall, 8 of 17 patients (47.1\%) experienced complete initial relief following the third GKRS. Of note, only 3 of these patients reported complete initial pain relief after primary GKRS, while 6 patients reported this following the second. The mean cumulative dose received by the 8 patients who experienced initial complete pain relief following the third GKRS was 195 Gy. Interestingly, the mean cumulative dose in the remaining nine patients was 220.5 Gy (BNI Scores II-V). This observation appears to contradict the expectation that higher doses lead to greater pain relief, but it must be viewed in context. Each of the 17 patients undergoing 3 separate GKRS procedures was subject to a steep elevation in his/her cumulative radiation dose with each repeat GKRS. There may exist a threshold cumulative dose beyond which the rate of pain relief plateaus or even falls. ${ }^{1,9,24}$ It must be noted that despite the median cumulative target dose of $210 \mathrm{~Gy}$, there were no adverse brainstem injuries in our series; it appears that optimal target selection can help to avoid pontine injury. Figure 3 demonstrates the lack of signal changes in the brainstem with the increasing cumulative radiation to the brainstem margin. Figure 4 is a composite depicting the target selection in a single patient and the dose histogram received by the brainstem at each of the first, second, and third radiosurgical treatments.

The 17 patients in our series experienced recurrent TN after a second radiosurgical procedure at a mean of 34.8 months (range 3.0-72.0 months). Every patient experienced acceptable initial pain relief after both their initial GKRS (including 6 patients who experienced complete pain relief) and second GKRS (8 patients experienced complete relief). It has been suggested that patients who experience complete pain relief after primary GKRS and experience recurrent TN in a reduced trigeminal distribution have the greatest chance at initial adequate pain relief after repeat GKRS. ${ }^{13,18}$ In our series, 6 patients experienced recurrent $\mathrm{TN}$ in a distribution different from that at the original presentation or after the first or second radiosurgical procedures; in 2 patients, this was a reduced distribution. Consistent with literature findings, repeat GKRS achieved complete initial pain relief from TN in both of these patients.

At a mean follow-up of 22.9 months following the third radiosurgical procedure, 6 patients $(35.3 \%)$ continued to report complete pain relief (BNI Score I); 7 others (41.2\%) reported incomplete but satisfactory pain relief (BNI Scores II-IIIb). This high rate of at least adequate pain relief, in $76.5 \%$ of patients, is comparable to pain outcomes described for repeat GKRS for TN. In addition, the time to initial response did not significantly differ in our series from the first to the second or the third radiosurgical procedure, averaging 2.9 months after both the repeat procedures. Initial pain relief after the second GKRS has been reported to occur at a median of approximately 3 months. ${ }^{15,34,46}$

\section{Pain Recurrence Despite 3 GKRS Procedures}

Four patients developed recurrent TN (BNI Score IV) by the time of last follow-up after the third GKRS. This rate of $23.5 \%$ at an average interval of approximately 2 years (mean 19.1 months among those who developed recurrent TN) is similar to the rates reported for initial repeat GKRS in the literature, with recurrence rates ranging from $11 \%$ to $38 \%$ at a mean interval of 18.6 months. Pain relief after the initial repeat GKRS in our series lasted an average of 34.8 months. Two patients who developed recurrences reported an initial BNI pain score of I following the third GKRS, while in the other 2 patients, one reported a BNI score of II and the other a BNI score of IIIa. All 4 patients had typical TN. One of these 4 patients underwent 2 PRGR procedures following the second radiosurgical procedure, but otherwise the 4 patients with
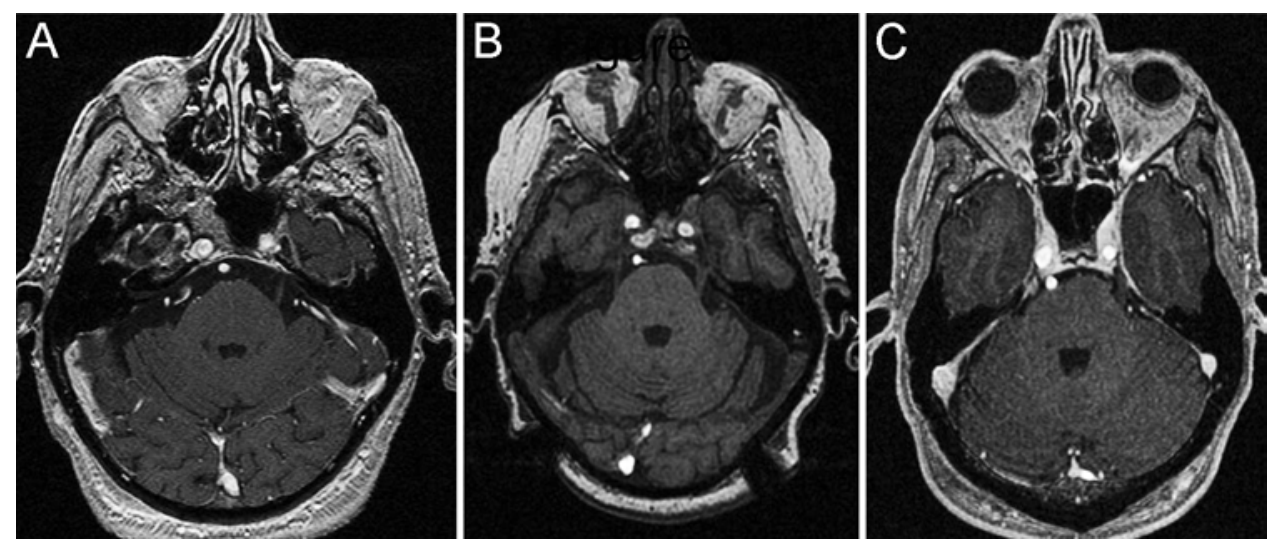

FIG. 3. Axial T1-weighted MR images with contrast obtained in 3 representative patients at the time of the third GKRS procedure, demonstrating an absence of adverse signal changes in the brainstem in a 96-year-old man who underwent GKRS for right-sided V2 and V3 TN (A), an 88-year-old woman who underwent GKRS for right-sided V2 and V3 TN (B), and a 52-year-old man who underwent GKRS for right-sided V2 TN (C). Although the ipsilateral trigeminal nerve is mildly hyperintense in all cases, especially in $\mathrm{A}$ and $\mathrm{C}$, no abnormalities in the brainstem are evident. 


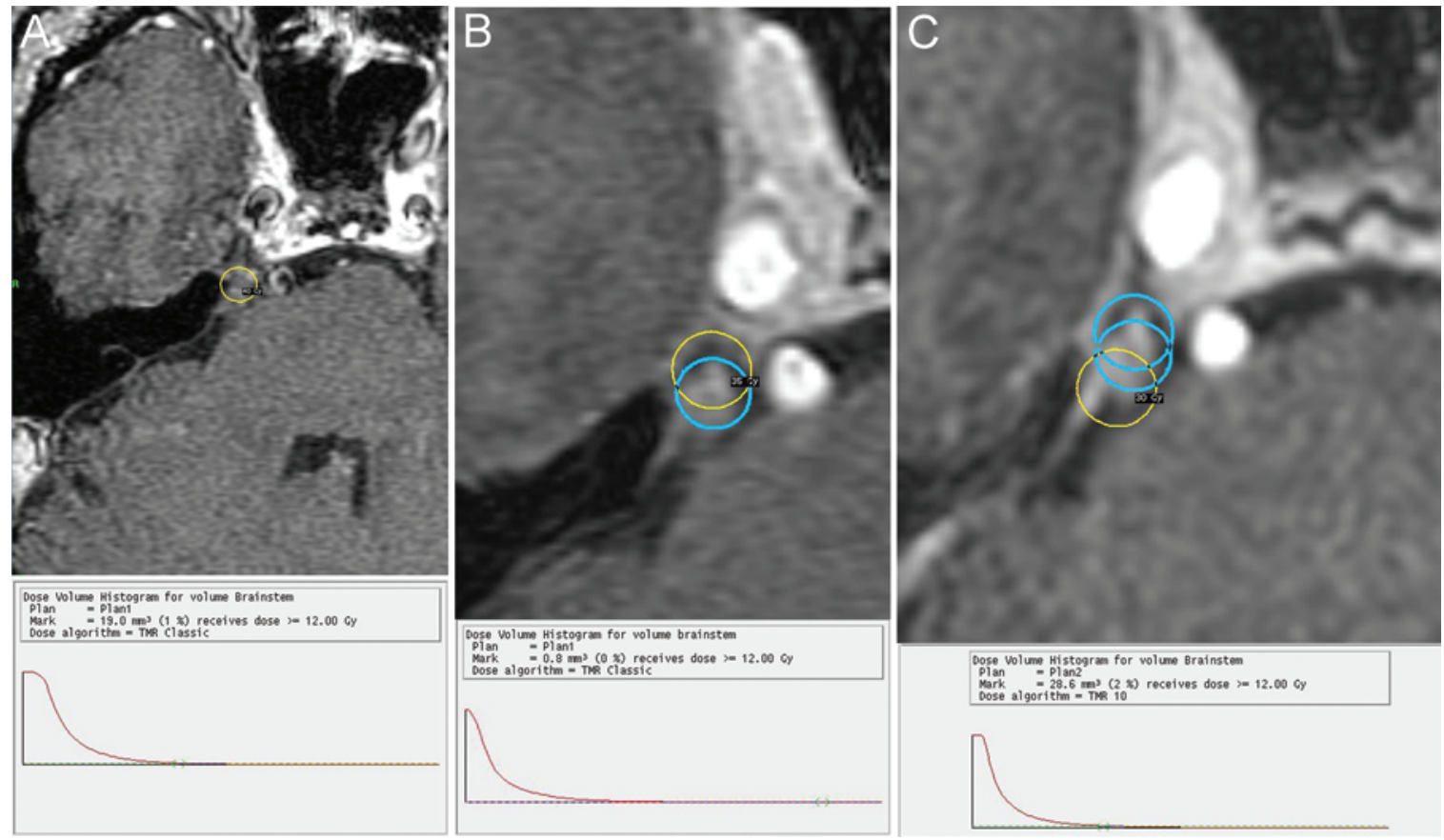

FIG. 4. T1-weighted MR images with contrast, obtained in a patient (an 88-year-old woman who underwent GKRS for right-sided V2 and V3 TN), demonstrating the target volume at each of the first (A), second (B), and third (C) GKRS procedures. Below each MR image the dose histogram is shown, demonstrating minimal radiation received by the brainstem in each of the 3 radiosurgical treatments. Figure is available in color online only.

recurrent TN (BNI Score IV or V) did not differ in demographics or treatment characteristics from the others at the time of the third GKRS.

Second repeat GKRS for medically refractory TN appears to be a promising treatment modality especially in elderly patients or those who may be poor surgical candidates due to coexisting comorbidities. This study is a retrospective analysis of patients who underwent a third GKRS procedure for intractable TN and, as such, is subject to all retrospective biases of clinical research. This study is not suitable to offer insights regarding practice guidelines or to make suggestions regarding optimal treatment options for patients. Prospective, randomized, well-controlled trials are necessary to fully evaluate and accurately assess the best form of treatment for patients with recurrent, intractable TN in whom other therapeutic modalities have failed, but these trials may not be feasible because of small patient numbers and the need for individualization based on prior treatment and factors affecting suitability for various treatment options. All patients included in our study were expected to benefit from a third GKRS because of their uniformly favorable responses to both first and second radiosurgical procedures (relief to at least BNI Scores I-IIIb). From our preliminary experience presented in this report, then, it appears that a third GKRS procedure may be best suited for patients who are poor candidates for alternative therapies because they: 1) are of advanced age and/or have severe general medical comorbidities, 2) are on a regimen of anticoagulation medication, 3) have undergone prior surgical procedures that have failed, or 4) continue to experience intractable pain despite maximal and varied medical therapy.

It is important to understand some key limitations of our study. To our knowledge, this represents the largest study sample to date. Analyses of safety and efficacy of multiple GKRS procedures have been limited thus far to those of single repeat GKRS only. Consequently, comparisons that we wished to make between our results and those in the literature, such as extent or duration of pain relief and rate of complications, were forced to be made with studies based on single repeat GKRS for TN, or studies of alternate treatment options for recurrent, intractable $\mathrm{TN}$. We recognize that this is not ideal, but in the absence of a robust body of literature built around double repeat GKRS for TN, this is the best comparison available. It is unfortunate that the small population of individuals who would be candidates for a third GKRS procedure prevents rapid accumulation of evidence upon which to base future practice. Although this report may suffice for a preliminary summary of outcomes and a review of triple GKRS for TN, future prospective, randomized controlled trials would be ideal to more precisely determine the optimal treatment modality for medically refractory, recurrent $\mathrm{TN}$, and, as stated above, to offer practice guidelines.

\section{Conclusions}

Gamma Knife radiosurgery is the most minimally invasive management option for patients with medically refractory TN. It is feasible in most patients, regardless of age or medical condition, including patients receiving anticoagulation or antiplatelet therapy. It has found success for recurrent TN as well, and, as we found in our analyses, GKRS appears to be a safe, moderately effective and long-lasting management tool for recurrent and refractory TN. No new instances of trigeminal sensory 
dysfunction were noted following the third GKRS, even after an extended follow-up. No patient suffered a pontine injury, developed diplopia, corneal anesthesia, or experienced a delayed vascular ischemic event. Key variables to optimizing treatment outcomes with GKRS may be cumulative dose selection, target selection, and timing from prior GKRS. The versatility in applicability of GKRS to non- or poor open surgical candidates and its successes in the treatment of recurrent $\mathrm{TN}$, as we discovered in our series, suggest that triple GKRS may offer an important tool in the management of medically refractory, recurrent TN especially in patients that have had good outcomes with prior radiosurgical procedures.

\section{Acknowledgment}

We wish to thank Sharon DeCesare, Regulatory Coordinator, University of Pittsburgh Medical Center, NAGKC, for her tireless efforts in obtaining and processing data from participating centers in the NAGKC, thereby enabling this study.

\section{References}

1. Aubuchon AC, Chan MD, Lovato JF, Balamucki CJ, Ellis TL, Tatter SB, et al: Repeat gamma knife radiosurgery for trigeminal neuralgia. Int J Radiat Oncol Biol Phys 81:1059-1065, 2011

2. Brisman R: Gamma knife radiosurgery for primary management for trigeminal neuralgia. J Neurosurg 93 Suppl 3:159-161, 2000

3. Brisman R: Repeat gamma knife radiosurgery for trigeminal neuralgia. Stereotact Funct Neurosurg 81:43-49, 2003

4. Burchiel KJ: A new classification for facial pain. Neurosurgery 53:1164-1167, 2003

5. Chang JW, Chang JH, Park YG, Chung SS: Gamma knife radiosurgery for idiopathic and secondary trigeminal neuralgia. J Neurosurg 93 Suppl 3:147-151, 2000

6. Dhople AA, Adams JR, Maggio WW, Naqvi SA, Regine WF, Kwok Y: Long-term outcomes of Gamma Knife radiosurgery for classic trigeminal neuralgia: implications of treatment and critical review of the literature. Clinical article. J Neurosurg 111:351-358, 2009

7. Dieckmann G, Bockermann V, Heyer C, Henning J, Roesen M: Five-and-a-half years' experience with percutaneous retrogasserian glycerol rhizotomy in treatment of trigeminal neuralgia. Appl Neurophysiol 50:401-413, 1987

8. Drzymala RE, Malyapa RS, Dowling JL, Rich KM, Simpson JR, Mansur DB: Gamma knife radiosurgery for trigeminal neuralgia: the Washington University initial experience. Stereotact Funct Neurosurg 83:148-152, 2005

9. Dvorak T, Finn A, Price LL, Mignano JE, Fitzek MM, Wu JK, et al: Retreatment of trigeminal neuralgia with Gamma Knife radiosurgery: is there an appropriate cumulative dose? Clinical article. J Neurosurg 111:359-364, 2009

10. Elias WJ, Burchiel KJ: Trigeminal neuralgia and other craniofacial pain syndromes: an overview. Semin Neurosurg 15:59-69, 2004

11. Eller JL, Raslan AM, Burchiel KJ: Trigeminal neuralgia: definition and classification. Neurosurg Focus 18(5):E3, 2005

12. Fountas KN, Lee GP, Smith JR: Outcome of patients undergoing gamma knife stereotactic radiosurgery for medically refractory idiopathic trigeminal neuralgia: Medical College of Georgia's experience. Stereotact Funct Neurosurg 84:88-96, 2006

13. Gellner V, Kurschel S, Kreil W, Holl EM, Ofner-Kopeinig P, Unger F: Recurrent trigeminal neuralgia: long term outcome of repeat gamma knife radiosurgery. J Neurol Neurosurg Psychiatry 79:1405-1407, 2008
14. Gorgulho AA, De Salles AAF: Impact of radiosurgery on the surgical treatment of trigeminal neuralgia. Surg Neurol 66:350-356, 2006

15. Han JH, Kim DG, Chung HT, Paek SH, Kim YH, Kim CY, et al: Long-term outcome of gamma knife radiosurgery for treatment of typical trigeminal neuralgia. Int J Radiat Oncol Biol Phys 75:822-827, 2009

16. Hasegawa T, Kondziolka D, Spiro R, Flickinger JC, Lunsford LD: Repeat radiosurgery for refractory trigeminal neuralgia. Neurosurgery 50:494-502, 2002

17. Henson CF, Goldman HW, Rosenwasser RH, Downes MB, Bednarz G, Pequignot EC, et al: Glycerol rhizotomy versus gamma knife radiosurgery for the treatment of trigeminal neuralgia: an analysis of patients treated at one institution. Int J Radiat Oncol Biol Phys 63:82-90, 2005

18. Herman JM, Petit JH, Amin P, Kwok Y, Dutta PR, Chin LS: Repeat gamma knife radiosurgery for refractory or recurrent trigeminal neuralgia: treatment outcomes and quality-of-life assessment. Int J Radiat Oncol Biol Phys 59:112-116, 2004

19. Huang CF, Chuang JC, Tu HT, Lin LY: Repeated Gamma Knife surgery for refractory trigeminal neuralgia. J Neurosurg 105 Suppl:99-102, 2006

20. Huang CF, Tu HT, Liu WS, Chiou SY, Lin LY: Gamma Knife surgery used as primary and repeated treatment for idiopathic trigeminal neuralgia. J Neurosurg 109 Suppl:179-184, 2008

21. Kano H, Kondziolka D, Mathieu D, Stafford SL, Flannery TJ, Niranjan A, et al: Stereotactic radiosurgery for intractable cluster headache: an initial report from the North American Gamma Knife Consortium. Clinical article. J Neurosurg 114:1736-1743, 2011

22. Kano H, Kondziolka D, Yang HC, Zorro O, Lobato-Polo J, Flannery TJ, et al: Outcome predictors after gamma knife radiosurgery for recurrent trigeminal neuralgia. Neurosurgery 67:1637-1645, 2010

23. Kanpolat Y, Savas A, Bekar A, Berk C: Percutaneous controlled radiofrequency trigeminal rhizotomy for the treatment of idiopathic trigeminal neuralgia: 25-year experience with 1,600 patients. Neurosurgery 48:524-534, 2001

24. Kimball BY, Sorenson JM, Cunningham D: Repeat Gamma Knife surgery for trigeminal neuralgia: long-term results. Clinical article. J Neurosurg 113 Suppl:178-183, 2010

25. Kondziolka D, Lacomis D, Niranjan A, Mori Y, Maesawa S, Fellows W, et al: Histological effects of trigeminal nerve radiosurgery in a primate model: implications for trigeminal neuralgia radiosurgery. Neurosurgery 46:971-977, 2000

26. Kondziolka D, Lunsford LD, Flickinger JC, Young RF, Vermeulen S, Duma CM, et al: Stereotactic radiosurgery for trigeminal neuralgia: a multiinstitutional study using the gamma unit. J Neurosurg 84:940-945, 1996

27. Kondziolka D, Zorro O, Lobato-Polo J, Kano H, Flannery TJ, Flickinger JC, et al: Gamma Knife stereotactic radiosurgery for idiopathic trigeminal neuralgia. Clinical article. J Neurosurg 112:758-765, 2010

28. Latchaw JP Jr, Hardy RW Jr, Forsythe SB, Cook AF: Trigeminal neuralgia treated by radiofrequency coagulation. $\mathbf{J}$ Neurosurg 59:479-484, 1983

29. Lim M, Villavicencio AT, Burneikiene S, Chang SD, Romanelli P, McNeely L, et al: CyberKnife radiosurgery for idiopathic trigeminal neuralgia. Neurosurg Focus 18(5):E9, 2005

30. Maesawa S, Salame C, Flickinger JC, Pirris S, Kondziolka D, Lunsford LD: Clinical outcomes after stereotactic radiosurgery for idiopathic trigeminal neuralgia. J Neurosurg 94:14-20, 2001

31. Menzel J, Piotrowski W, Penzholz H: Long-term results of Gasserian ganglion electrocoagulation. J Neurosurg 42:140143,1975

32. Mohammad-Mohammadi A, Recinos PF, Lee JH, Elson P, 
Barnett GH: Surgical outcomes of trigeminal neuralgia in patients with multiple sclerosis. Neurosurgery 73:941-950, 2013

33. Oturai AB, Jensen K, Eriksen J, Madsen F: Neurosurgery for trigeminal neuralgia: comparison of alcohol block, neurectomy, and radiofrequency coagulation. Clin J Pain 12:311-315, 1996

34. Park KJ, Kondziolka D, Berkowitz O, Kano H, Novotny J Jr, Niranjan A, et al: Repeat gamma knife radiosurgery for trigeminal neuralgia. Neurosurgery 70:295-305, 2012

35. Pollock BE: Percutaneous retrogasserian glycerol rhizotomy for patients with idiopathic trigeminal neuralgia: a prospective analysis of factors related to pain relief. J Neurosurg 102:223-228, 2005

36. Pollock BE, Foote RL, Link MJ, Stafford SL, Brown PD, Schomberg PJ: Repeat radiosurgery for idiopathic trigeminal neuralgia. Int J Radiat Oncol Biol Phys 61:192-195, 2005

37. Pollock BE, Foote RL, Stafford SL, Link MJ, Gorman DA, Schomberg PJ: Results of repeated gamma knife radiosurgery for medically unresponsive trigeminal neuralgia. J Neurosurg 93 Suppl 3:162-164, 2000

38. Pollock BE, Phuong LK, Foote RL, Stafford SL, Gorman DA: High-dose trigeminal neuralgia radiosurgery associated with increased risk of trigeminal nerve dysfunction. Neurosurgery 49:58-64, 2001

39. Pollock BE, Phuong LK, Gorman DA, Foote RL, Stafford SL: Stereotactic radiosurgery for idiopathic trigeminal neuralgia. J Neurosurg 97:347-353, 2002

40. Pollock BE, Stein KJ: Surgical management of trigeminal neuralgia patients with recurrent or persistent pain despite three or more prior operations. World Neurosurg 73:523528,2010

41. Régis J, Metellus P, Hayashi M, Roussel P, Donnet A, BilleTurc F: Prospective controlled trial of gamma knife surgery for essential trigeminal neuralgia. J Neurosurg 104:913924, 2006

42. Riesenburger RI, Hwang SW, Schirmer CM, Zerris V, Wu JK, Mahn K, et al: Outcomes following single-treatment Gamma Knife surgery for trigeminal neuralgia with a minimum 3-year follow-up. Clinical article. J Neurosurg 112:766-771, 2010

43. Rogers CL, Shetter AG, Fiedler JA, Smith KA, Han PP, Speiser BL: Gamma knife radiosurgery for trigeminal neuralgia: the initial experience of The Barrow Neurological Institute. Int J Radiat Oncol Biol Phys 47:1013-1019, 2000

44. Sheehan J, Pan HC, Stroila M, Steiner L: Gamma knife surgery for trigeminal neuralgia: outcomes and prognostic factors. J Neurosurg 102:434-441, 2005
45. Shetter AG, Rogers CL, Ponce F, Fiedler JA, Smith K, Speiser BL: Gamma knife radiosurgery for recurrent trigeminal neuralgia. J Neurosurg 97 (5 Suppl):536-538, 2002

46. Urgosik D, Liscak R, Novotny J Jr, Vymazal J, Vladyka V: Treatment of essential trigeminal neuralgia with gamma knife surgery. J Neurosurg 102 Suppl:29-33, 2005

47. Verheul JB, Hanssens PEJ, Lie ST, Leenstra S, Piersma H, Beute GN: Gamma Knife surgery for trigeminal neuralgia: a review of 450 consecutive cases. Clinical article. J Neurosurg 113 Suppl:160-167, 2010

48. Villavicencio AT, Lim M, Burneikiene S, Romanelli P, Adler JR, McNeely L, et al: Cyberknife radiosurgery for trigeminal neuralgia treatment: a preliminary multicenter experience. Neurosurgery 62:647-655, 2008

49. Wang DD, Ouyang D, Englot DJ, Rolston JD, Molinaro AM, Ward M, et al: Trends in surgical treatment for trigeminal neuralgia in the United States of America from 1988 to 2008. J Clin Neurosci 20:1538-1545, 2013

50. Young B, Shivazad A, Kryscio RJ, St Clair W, Bush HM: Long-term outcome of high-dose Gamma Knife surgery in treatment of trigeminal neuralgia. Clinical article. J Neurosurg 119:1166-1175, 2013

\section{Author Contributions}

Conception and design: Lunsford, Tempel, Chivukula, Bowden, Kano, Niranjan, Kaufmann, Sheehan, Mathieu. Acquisition of data: Tempel, Chivukula, Bowden, Kano, Chang, Sneed, Kaufmann, Sheehan, Mathieu. Analysis and interpretation of data: Tempel, Chivukula, Chang, Kaufmann, Sheehan, Mathieu. Drafting the article: Tempel, Chivukula, Sneed. Critically revising the article: all authors. Reviewed submitted version of manuscript: all authors. Approved the final version of the manuscript on behalf of all authors: Lunsford. Statistical analysis: Chivukula. Administrative/technical/material support: Chivukula, Bowden, Niranjan, Chang, Kaufmann, Sheehan, Mathieu.

\section{Supplemental Information \\ Current Affiliation}

Dr. Chivukula: Department of Neurological Surgery, UCLA Health System, Los Angeles, CA.

\section{Correspondence}

L. Dade Lunsford, Department of Neurosurgery, University of Pittsburgh Medical Center, UPMC Presbyterian, 200 Lothrop St., Ste. B-400, Pittsburgh, PA 15213. email: lunsld@upmc.edu. 